\title{
A New Post-Processing Technique for Finite Element Methods with $L^{2}$-Superconvergence
}

\author{
Wei Pi, Hao Wang* and Xiaoping Xie \\ School of Mathematics, Sichuan University, No. 24 South Section One, Yihuan \\ Road, Chengdu 610065, China.
}

Received 17 January 2019; Accepted (in revised version) 20 May 2019.

\begin{abstract}
A simple post-processing technique for finite element methods with $L^{2}$-superconvergence is proposed. It provides more accurate approximations for solutions of twoand three-dimensional systems of partial differential equations. Approximate solutions can be constructed locally by using finite element approximations $u_{h}$ provided that $u_{h}$ is superconvergent for a locally defined projection $\widetilde{P}_{h} u$. The construction is based on the least-squares fitting algorithm and local $L^{2}$-projections. Error estimates are derived and numerical examples illustrate the effectiveness of this approach for finite element methods.
\end{abstract}

AMS subject classifications: 65N30, 65N15

Key words: Finite element method, post-processing, least-square fitting, $L^{2}$-superconvergence.

\section{Introduction}

The post-processing of approximate solutions is a commonly used procedure to obtain more accurate approximations for important quantities in numerical methods for partial differential equations [4-6,22,23]. Post-processing or/and recovery techniques have been developed for plenty of finite element methods with superconvergence $[1,7,8,10$, $12,13,15,18,20]$. In particular, for the Raviart-Thomas and Brezzi-Douglas-Marini mixed elements methods for second order elliptic problems, the post-processed approximations with improved accuracy are constructed via element-by-element solution of local problems with respect to the finite element solutions of the scalar variable and the Lagrange multiplier $[1,8]$. In contrast to the post-processing methods $[1,8]$, Stenberg [18] proposed an approach based on solving local problems with respect to the mixed finite element approximations of the scalar variable and its gradient. Following ideas of [18], Cockburn et al. $[12,13]$ developed an element-by-element post-processing of the scalar variable for the elliptic problems and velocity variable in the Stokes problem for HDG methods.

*Corresponding author. Email addresses: 512442593@qq.com (W. Pi), wangh@scu.edu.cn (H. Wang), xpxie@scu.edu.cn (X. Xie) 
Bramble and $\mathrm{Xu}$ [7] proposed a general post-processing technique for various mixed finite element methods with the superconvergence estimate

$$
\left\|\widetilde{P}_{h} u-u_{h}\right\|_{L^{p}(\Omega)} \leq C h^{k+2}|\log h|^{\mu_{1}}
$$

and the gradient approximation estimate

$$
\left\|\nabla u-(\nabla u)_{h}\right\|_{L^{p}(\Omega)} \leq C h^{k+1}|\log h|^{\mu_{2}},
$$

where $u$ is the exact solution of a system of partial differential equations on a domain $\Omega \subset \Re^{2}, C>0$ a generic constant, which depends on $u$ but not on the mesh size $h ; \mu_{1}, \mu_{2}$ are nonnegative constants and $u_{h} \in W_{h}$ and $(\nabla u)_{h} \in V_{h}$ are finite element approximations of $u$ and $\nabla u$, respectively. Moreover, $W_{h}$ and $V_{h}$ are finite-dimensional subspaces of $L^{p}(\Omega)$, $p \geq 1, W_{h}$ consists of discontinuous piecewise polynomials of degree at most $k \geq 0$, and $\widetilde{P}_{h}$ is a locally defined operator, which is invariant on polynomials of degree $k$. Under a regularity condition for $u$, the post-processed approximation $u_{h}^{*}$ obtained from $u_{h}$ and $(\nabla u)_{h}$, satisfies the estimate

$$
\left\|u-u_{h}^{*}\right\|_{L^{p}(\Omega)} \leq C\left(\left\|\widetilde{P}_{h} u-u_{h}\right\|_{L^{p}(\Omega)}+h\left\|\nabla u-(\nabla u)_{h}\right\|_{L^{p}(\Omega)}+h^{k+2}\right) .
$$

Further, Zienkiewicz and Zhu [22,23] used the well-known gradient recovery technique, usually referred to as superconvergence patch recovery (SPR), to post-process the gradient $\nabla u_{h}$ of the finite element solution $u_{h}$. They constructed an SPR-recovered gradient by a local discrete least-squares fitting of polynomials of degree $k$ to the gradient values at sampling points on element patches. The superconvergence properties of this technique was discussed in Refs. [14,19,21]. Zhang and Naga [20] introduced a different gradient recovery method called the polynomial preserving recovery (PPR). To determine a recovered gradient, the method uses the least-squares algorithm to assign a polynomial of degree $k+1$ to the solution at chosen nodal points and computes the corresponding partial derivatives. Under certain conditions, the PPR post-processed gradient $G_{h} u_{h}$ satisfies the superconvergence estimate

$$
\left\|\nabla u-G_{h} u_{h}\right\|_{L^{\infty}\left(\Omega_{0}\right)} \leq C\left(h^{k+1}|\log h|^{\bar{r}}+h^{k+\sigma}\right),
$$

where $\sigma$ is a positive constant, $\Omega_{0} \subset \subset \Omega, \bar{r}=1$ if $k=1$ and $\bar{r}=0$ if $k \geq 2$.

However, to the best of authors' knowledge, there is no post-processing technique, which uses only $u_{h}$ to construct a superconvergent post-processed approximation $u_{h}^{*}$. Here, we present a general post-processing technique for direct construction of the improved approximation of $u$. The method is based on the least-squares algorithm and the local $L^{2}$-projection to determine a fitting polynomial from the finite element solution $u_{h}$. Our analysis depends only on a superconvergence result similar to (1.1) and the main result is proved in general approximation-theoretic settings. Therefore, its application is not restricted to the above mentioned finite element methods.

The rest of the paper is organised as follows. Section 2 contains necessary notations. Section 3 is devoted to the construction of the post-processed approximation, the error estimation, and the verification of assumptions. Finally, numerical results in Section 4 are aimed to verify the performance of the post-processing method proposed. 


\section{Notations}

Let $\Omega$ be a bounded domain in $\mathbb{R}^{n}, n=2,3$. For any bounded domain $D \subset \mathbb{R}^{n}, n=2,3$ and a nonnegative integer $m$, we denote by $H^{m}(D)$ the usual $m$-order Sobolev space on $D$ and let $\|\cdot\|_{m, D}$ and $|\cdot|_{m, D}$ refer to the corresponding norm and semi-norm, respectively. In particular, $H^{0}(D)$ is the space of square integrable functions $L^{2}(D)$ with the inner product $(\cdot, \cdot)_{D}$ and the norm $\|\cdot\|_{0, D}$. If $D=\Omega$, we write $\|\cdot\|_{m}:=\|\cdot\|_{m, \Omega}$ and $|\cdot|_{m}:=|\cdot|_{m, \Omega}$. By $\mathscr{P}_{m}(D)$ we denote the set of all polynomials on $D$ of degree at most $m$.

Let $\mathscr{T}_{h}$ be a shape regular partition of the domain $\Omega$, which consists of closed polygons $T$ - cf. [11], with the mesh size $h=\max _{T \in \mathscr{T}_{h}} h_{T}$, where $h_{T}$ is the diameter of $T$. The partition $\mathscr{T}_{h}$ can be conforming or nonconforming, which allows hanging nodes. Let $\mathscr{N}_{h}=$ $\left\{\boldsymbol{x}_{\boldsymbol{i}}: i=1,2, \ldots, n_{h}\right\}$ be the set of all nodes of the partition $\mathscr{T}_{h}$. For any $\boldsymbol{x}_{\boldsymbol{i}} \in \mathscr{N}_{h}$, we denote by $h_{i}$ the length of the longest edge attached to $x_{i}$ and let $M_{i}$ be the patch defined by

$$
M_{i}=M_{i}(\alpha):=\bigcup_{T \in \mathscr{T}_{h}, T \subseteq B_{\alpha h_{i}}\left(x_{i}\right)} T,
$$

where $B_{\alpha h_{i}}\left(x_{i}\right), \alpha>0$ is the ball

$$
B_{\alpha h_{i}}\left(x_{i}\right):=\left\{x \in \Omega:\left|x-x_{i}\right| \leq \alpha h_{i}\right\} .
$$

If $n_{i}=n_{i}(\alpha)$ is the number of elements in the patch $M_{i}$, we set

$$
\mathbb{M}_{h}:=\left\{M_{i}: i=1,2, \ldots, n_{h}\right\} .
$$

For any $T \in \mathscr{T}_{h}$ and for any integer $j \geq 0$, let $P_{T}^{j}: L^{2}(T) \longrightarrow \mathscr{P}_{j}(T)$ be the usual $L^{2}$ orthogonal projection. Define $\mathrm{P}_{M_{i}}^{j}: L^{2}\left(M_{i}\right) \longrightarrow L^{2}\left(M_{i}\right)$, such that for any $v \in L^{2}\left(M_{i}\right)$ the relation

$$
\left.\left(\mathrm{P}_{M_{i}}^{j} v\right)\right|_{T}=P_{T}^{j}\left(\left.v\right|_{T}\right) \quad \text { for all } \quad T \in M_{i}
$$

holds.

Throughout this paper, the notation $a \lesssim b(a \gtrsim b)$ means that $a \leq C b(a \geq C b)$ with a constant $C$, which depends on $u$ but not on the mesh size $h$.

\section{Main Results}

Let us assume that every patch $M_{i} \in \mathbb{M}_{h}$ satisfies the following conditions.

Condition 3.1. For a given integer $k \geq 0$, there exists a nonnegative integer $j \leq k$, such that for any $q \in \mathscr{P}_{k+1}\left(M_{i}\right), M_{i} \in \mathbb{M}_{h}$, the inequality

$$
\|q\|_{0, M_{i}} \lesssim\left\|\mathrm{P}_{M_{i}}^{j} q\right\|_{0, M_{i}}
$$

holds. 
Condition 3.2. Any $v \in H^{k+2}\left(M_{i}\right), i=1,2, \ldots, n_{h}$ satisfies the inequality

$$
\inf _{w \in \mathscr{P}_{k+1}\left(M_{i}\right)}\|v-w\|_{0, M_{i}} \lesssim h^{k+2}|v|_{k+2, M_{i}} .
$$

Condition 3.1 yields that $M_{i}$ has enough elements. We note that if $\alpha_{1}>\alpha_{2}$, then $n_{i}\left(\alpha_{1}\right)>n_{i}\left(\alpha_{2}\right)$, where $n_{i}\left(\alpha_{i}\right)$ is the number of element patches in $M_{i}\left(\alpha_{j}\right), j=1,2$. Thus it is natural to choose the smallest $\alpha$ satisfying Condition 3.1. On the other hand, Condition 3.2 represents the standard approximation property.

Condition 3.1 allows us to equip $\mathscr{P}_{k+1}\left(M_{i}\right)$ with an inner product and a norm.

Lemma 3.1. For any $M_{i} \in \mathbb{M}_{h}$, the inner product and the norm on the space $\mathscr{P}_{k+1}\left(M_{i}\right)$ can be, respectively, defined as $\left(P_{M_{i}}^{j} \cdot P_{M_{i}}^{j}\right)_{M_{i}}$ and $\left\|P_{M_{i}}^{j} \cdot\right\|_{0, M_{i}}$.

Proof. Let us show that $\left(\mathrm{P}_{M_{i}}^{j}, \mathrm{P}_{M_{i}}^{j} \cdot\right)_{M_{i}}$ is an inner product on $\mathscr{P}_{k+1}\left(M_{i}\right)$. Recalling the relation (2.1), we only have to show that if $q \in \mathscr{P}_{k+1}\left(M_{i}\right)$ and

$$
\left(\mathrm{P}_{M_{i}}^{j} q, \mathrm{P}_{M_{i}}^{j} q\right)_{M_{i}}=0
$$

then $q=0$. However, the condition (3.1) yields

$$
\|q\|_{0, M_{i}}^{2} \lesssim\left\|\mathrm{P}_{M_{i}}^{j} q\right\|_{0, M_{i}}^{2}=\left(\mathrm{P}_{M_{i}}^{j} q, \mathrm{P}_{M_{i}}^{j} q\right)_{M_{i}}=0
$$

and the proof is completed.

\subsection{Recovery operator}

Definition 3.1. For any $M_{i} \in \mathbb{M}_{h}$, the local recovery operator $R_{M_{i}}: L^{2}\left(M_{i}\right) \rightarrow \mathscr{P}_{k+1}\left(M_{i}\right)$ is defined by the relations

$$
\left(\mathrm{P}_{M_{i}}^{j} R_{M_{i}} v, \mathrm{P}_{M_{i}}^{j} q\right)_{M_{i}}=\left(\mathrm{P}_{M_{i}}^{j} \nu, \mathrm{P}_{M_{i}}^{j} q\right)_{M_{i}} \quad \text { for all } \quad v \in L^{2}\left(M_{i}\right), q \in \mathscr{P}_{k+1}\left(M_{i}\right) .
$$

According to Lemma 3.1, the operator $R_{M_{i}}$ is well-defined. Moreover, the following result holds.

Lemma 3.2. For any $M_{i} \in \mathbb{M}_{h}$, the recovery operator $R_{M_{i}}$ is an orthogonal projection onto $\mathscr{P}_{k+1}\left(M_{i}\right)$ with respect to the inner product $\left(P_{M_{i}}^{j} \cdot P_{M_{i}}^{j} \cdot\right)_{M_{i}}$ and if $v \in L^{2}\left(M_{i}\right)$, then

$$
R_{M_{i}} v=\underset{q \in \mathscr{P}_{k+1}\left(M_{i}\right)}{\arg \min }\left\|P_{M_{i}}^{j}(v-q)\right\|_{0, M_{i}}
$$

Other consequences of Conditions 3.1 and the inequality 3.1 are presented in Lemmas 3.2-3.5. 
Lemma 3.3. For any $v \in L^{2}\left(M_{i}\right)$ and $M_{i} \in \mathbb{M}_{h}$ the inequalities

$$
\left\|R_{M_{i}} v\right\|_{0, M_{i}} \lesssim\left\|P_{M_{i}}^{j} v\right\|_{0, M_{i}} \lesssim\|v\|_{0, M_{i}}
$$

hold.

Lemma 3.4. For any $v \in H^{k+2}\left(M_{i}\right)$ and $M_{i} \in \mathbb{M}_{h}$, the inequality

$$
\left\|v-R_{M_{i}} v\right\|_{0, M_{i}} \lesssim h^{k+2}|v|_{k+2, M_{i}}
$$

holds.

Proof. Since $w=R_{M_{i}} w$ for any $w \in \mathscr{P}_{k+1}\left(M_{i}\right)$, Condition 3.2 and the inequality (3.2) lead to the estimate

$$
\begin{aligned}
\left\|v-R_{M_{i}} v\right\|_{0, M_{i}} & =\inf _{w \in \mathscr{P}_{k+1}\left(M_{i}\right)}\left\|v-w-R_{M_{i}}(v-w)\right\|_{0, M_{i}} \\
& \lesssim \inf _{w \in \mathscr{P}_{k+1}\left(M_{i}\right)}\|v-w\|_{0, M_{i}} \lesssim h^{k+2}|v|_{k+2, M_{i}}
\end{aligned}
$$

as required.

Let $\widetilde{P}_{h}$ be an operator defined on $L^{2}(\Omega)$, such that its restriction $\left.\widetilde{P}_{h}\right|_{T}: L^{2}(T) \longrightarrow L^{2}(T)$, $T \in M_{i}$ satisfies the conditions

$$
\left(\widetilde{P}_{h} w, v\right)_{T}=(w, v)_{T}
$$

valid for all $w \in L^{2}(T)$ and all $v \in \mathscr{P}_{j}(T)$.

Lemma 3.5. If $v \in L^{2}\left(M_{i}\right)$ and $M_{i} \in \mathbb{M}_{h}$, then

$$
R_{M_{i}} v=R_{M_{i}} \widetilde{P}_{h} v
$$

Proof. It follows from the definitions of $R_{M_{i}}, \mathrm{P}_{M_{i}}^{j}, P_{T}^{j}$ and the Eq. (3.3) that for $v \in L^{2}\left(M_{i}\right)$ and $q \in \mathscr{P}_{k+1}\left(M_{i}\right)$ one has

$$
\begin{aligned}
\left(\mathrm{P}_{M_{i}}^{j} R_{M_{i}} \widetilde{P}_{h} v, \mathrm{P}_{M_{i}}^{j} q\right)_{M_{i}} & =\left(\mathrm{P}_{M_{i}}^{j} \widetilde{P}_{h} v, \mathrm{P}_{M_{i}}^{j} q\right)_{M_{i}}=\sum_{T \in M_{i}}\left(P_{T}^{j} \widetilde{P}_{h} v, P_{T}^{j} q\right)_{M_{i}} \\
& =\sum_{T \in M_{i}}\left(\widetilde{P}_{h} v, P_{T}^{j} q\right)_{M_{i}}=\sum_{T \in M_{i}}\left(v, P_{T}^{j} q\right)_{M_{i}} \\
& =\sum_{T \in M_{i}}\left(P_{T}^{j} v, P_{T}^{j} q\right)_{M_{i}}=\left(\mathrm{P}_{M_{i}}^{j} v, \mathrm{P}_{M_{i}}^{j} q\right)_{M_{i}}=\left(\mathrm{P}_{M_{i}}^{j} R_{M_{i}} v, \mathrm{P}_{M_{i}}^{j} q\right)_{M_{i}},
\end{aligned}
$$

which yields the representation (3.4). 


\subsection{Post-processed approximation}

For any $T \in \mathscr{T}_{h}$, we set

$$
\mathbb{M}_{T}:=\left\{M_{i} \in \mathbb{M}_{h}: T \in M_{i}\right\},
$$

where $n_{T}$ is the number of element patches in $\mathbb{M}_{T}$.

Let $u_{h} \in L^{2}(\Omega)$ be a finite element approximation of $u$, such that

$$
\left\|u-u_{h}\right\|_{0} \lesssim h^{r}, \quad r \leq k+1 .
$$

Considering a post-processed approximation $u_{h}^{*}$ defined by

$$
\left.u_{h}^{*}\right|_{T}=\left.\sum_{M_{i} \in \mathbb{M}_{T}} \frac{1}{n_{T}}\left(R_{M_{i}} u_{h}\right)\right|_{T}, \quad T \in \mathscr{T}_{h},
$$

and using Lemmas 3.3, 3.4 and 3.5, we obtain the following results.

Theorem 3.1. If $\widetilde{P}_{h}$ satisfies the projection property (3.3) and $u \in H^{k+2}(\Omega)$, then

$$
\left\|u-u_{h}^{*}\right\|_{0} \lesssim\left\|\widetilde{P}_{h} u-u_{h}\right\|_{0}+h^{k+2}|u|_{k+2} .
$$

Moreover, if

$$
\left\|\widetilde{P}_{h} u-u_{h}\right\|_{0} \lesssim h^{k+2}|u|_{k+2}
$$

then the superconvergence estimate

$$
\left\|u-u_{h}^{*}\right\|_{0} \lesssim h^{k+2}|u|_{k+2}
$$

holds.

Proof. It follows from (3.5) that

$$
\begin{aligned}
\left\|u-u_{h}^{*}\right\|_{0}^{2} & =\sum_{T \in \mathscr{T}_{h}}\left\|u-u_{h}^{*}\right\|_{0, T}^{2} \leq \sum_{M_{i} \in \mathbb{M}_{h}} \sum_{T \in M_{i}}\left\|u-\sum_{M_{j} \in \mathbb{M}_{T}} \frac{1}{n_{T}} R_{M_{j}} u_{h}\right\|_{0, T}^{2} \\
& =\sum_{M_{i} \in \mathbb{M}_{h}} \sum_{T \in M_{i}}\left\|\sum_{M_{j} \in \mathbb{M}_{T}} \frac{1}{n_{T}}\left(u-R_{M_{j}} u_{h}\right)\right\|_{0, T}^{2} \lesssim \sum_{M_{i} \in \mathbb{M}_{h}}\left\|u-R_{M_{i}} u_{h}\right\|_{0, M_{i}}^{2} .
\end{aligned}
$$

Using triangle inequality and Lemmas $3.3,3.4$ and 3.5, we obtain

$$
\begin{aligned}
\left\|u-R_{M_{i}} u_{h}\right\|_{0, M_{i}} & \lesssim\left\|u-R_{M_{i}} u\right\|_{0, M_{i}}+\left\|R_{M_{i}} u-R_{M_{i}} u_{h}\right\|_{0, M_{i}} \\
& \lesssim\left\|u-R_{M_{i}} u\right\|_{0, M_{i}}+\left\|R_{M_{i}}\left(\widetilde{P}_{h} u-u_{h}\right)\right\|_{0, M_{i}} \\
& \lesssim h^{k+2}|u|_{k+2, M_{i}}+\left\|\widetilde{P}_{h} u-u_{h}\right\|_{0, M_{i}} .
\end{aligned}
$$

This and (3.9) first yield (3.6) and consequently (3.8). 
Remark 3.1. Theorem 3.1 can be applied to various finite element methods, including the Raviart-Thomas triangular elements $\mathbf{R T}_{k}$ and rectangular elements $\mathbf{R T}_{[k]}$ with $k \geq 0$, the Brezzi-Douglas-Marini triangular elements $\mathbf{B D M}_{k}$ and rectangular elements $\mathbf{B D M}_{[k]}$ with $k \geq 2$, the PEERS elements, the mixed elements by Stenberg, the hybridised Discontinuous Galerkin triangular elements $\mathbf{H D G}_{k}$ and rectangular elements $\mathbf{H D G}_{[k]}$ with $k \geq 1-$ cf. Refs. $[1,2,8,9,12,17]$. Let us note the following properties of the above listed $2 D$ elements:

(1) $\mathbf{R T}_{k}$ elements $(k \geq 0):\left.u_{h}\right|_{T} \in \mathscr{P}_{k}(T)$ and $\left.\widetilde{P}_{h}\right|_{T}: L^{2}(T) \longrightarrow \mathscr{P}_{k}(T)$ is the $L^{2}$-orthogonal projection satisfying the projection property (3.3) for any $j \leq k$ and the superconvergence estimate (3.7).

(2) $\mathbf{R T}_{[k]}$ elements $(k \geq 0):\left.u_{h}\right|_{T} \in \mathscr{Q}_{k}(T)$ and $\left.\widetilde{P}_{h}\right|_{T}: L^{2}(T) \longrightarrow Q_{k}(T)$ is the $L^{2}$ orthogonal projection satisfying (3.3) (with any $j \leq k$ ) and (3.7). Here $Q_{k}(T)$ denotes the set of all polynomials on $T$ of degree at most $k$ in each variable.

(3) $\mathbf{B D M}_{k}$ and $\mathbf{B D M}_{[k]}$ elements $(k \geq 2):\left.u_{h}\right|_{T} \in \mathscr{P}_{k-1}(T)$ and $\left.\widetilde{P}_{h}\right|_{T}: L^{2}(T) \longrightarrow \mathscr{P}_{k-1}(T)$ is the $L^{2}$-orthogonal projection satisfying (3.3) for any $j \leq k-1$ and (3.7).

(4) PEERS elements: $\left.u_{h}\right|_{T} \in \mathscr{P}_{0}(T)$ and $\left.\widetilde{P}_{h}\right|_{T}: L^{2}(T) \longrightarrow \mathscr{P}_{0}(T)$ is the $L^{2}$-orthogonal projection satisfying (3.3) for $j=0$ and (3.7) with $k=0$.

(5) The mixed elements by Stenberg $(k \geq 1):\left.u_{h}\right|_{T} \in \mathscr{P}_{k-1}(T)$ and $\left.\widetilde{P}_{h}\right|_{T}: L^{2}(T) \longrightarrow$ $\mathscr{P}_{k-1}(T)$ is the $L^{2}$-orthogonal projection satisfying (3.3) for any $j \leq k-1$ and (3.7).

(6) HDG $_{k}$ and $\mathbf{H D G}_{[k]}$ elements $(k \geq 1):\left.u_{h}\right|_{T} \in \mathscr{P}_{k}(T)$ and $\left.\widetilde{P}_{h}\right|_{T}: L^{2}(T) \longrightarrow \mathscr{P}_{k}(T)$ is an operator satisfying (3.3) for $j=k-1$ and (3.7).

\subsection{Discussion on Condition 3.1}

As shown in Subsection 3.2, Condition 3.1 is crucial for the construction and evaluation of the post-processed approximation $u_{h}^{*}$. However, for a given $j$, Condition 3.1 requires the availability of sufficiently large number of elements $n_{i}$ in $M_{i}$.

Theorem 3.2. If the inequality (3.1) holds for any $q \in \mathscr{P}_{k+1}\left(M_{i}\right), M_{i} \in \mathbb{M}_{h}$, then

$$
n_{i} \geq \frac{C_{k+1+n}^{n}}{C_{j+n}^{n}}
$$

where $n=2$ or $n=3$ is the space dimension and $C_{l+n}^{n}=(l+n) ! /(l ! n !)$.

Proof. Since

$$
\mathscr{P}_{l}\left(M_{i}\right)=\operatorname{span}\left\{1, x_{1}, x_{2}, \ldots, x_{n}, x_{1}^{2}, x_{1} x_{2}, \ldots, x_{n}^{2}, x_{1}^{l}, x_{1}^{l-1} x_{2}, \ldots, x_{n}^{l}\right\},
$$

we can represent any $q \in \mathscr{P}_{k+1}\left(M_{i}\right)$ in the form

$$
q=\mathbf{P a},
$$


where

$$
\begin{aligned}
& \mathbf{P}:=\left(1, x_{1}, x_{2}, \ldots, x_{n}, x_{1}^{2}, x_{1} x_{2}, \ldots, x_{n}^{2}, x_{1}^{k+1}, x_{1}^{k} x_{2}, \ldots, x_{n}^{k+1}\right), \\
& \mathbf{a}=\left(a_{1}, a_{2}, \ldots, a_{\gamma}\right)^{T}, \quad \gamma=\operatorname{dim}\left(P_{k+1}\right)=C_{k+1+n}^{n} .
\end{aligned}
$$

It follows from (3.1) that if $P_{T}^{j} q=0$ for all $T \in M_{i}$, then $q=0$. Along with the definition of the projection $P_{T}^{j}$, this means that if the relation

$$
(q, v)_{T}=\left(P_{T}^{j} q, v\right)_{T}=0
$$

holds for any $v \in P_{j}(T), T \in M_{i}$, then $q=0$. Setting $M_{i}=\left\{T_{l}: l=1,2, \ldots, n_{i}\right\}$, we obtain that if (3.10) holds, then the condition

$$
A \mathbf{a}=0,
$$

where $A=\left(A_{1}, A_{2}, \ldots, A_{n_{i}}\right)^{T}$ and

$$
A_{l}=\left(\begin{array}{ccccccc}
(1,1)_{T_{l}} & \left(1, x_{1}\right)_{T_{l}} & \ldots & \left(1, x_{n}\right)_{T_{l}} & \left(1, x_{1}^{2}\right)_{T_{l}} & \ldots & \left(1, x_{n}^{k+1}\right)_{T_{l}} \\
\left(x_{1}, 1\right)_{T_{l}} & \left(x_{1}, x_{1}\right)_{T_{l}} & \ldots & \left(x_{1}, x_{n}\right)_{T_{l}} & \left(x_{1}, x_{1}^{2}\right)_{T_{l}} & \ldots & \left(x_{1}, x_{n}^{k+1}\right)_{T_{l}} \\
\vdots & \vdots & \vdots & \vdots & \vdots & \vdots & \vdots \\
\left(x_{n}, 1\right)_{T_{l}} & \left(x_{n}, x_{1}\right)_{T_{l}} & \ldots & \left(x_{n}, x_{n}\right)_{T_{l}} & \left(x_{n}, x_{1}^{2}\right)_{T_{l}} & \ldots & \left(x_{n}, x_{n}^{k+1}\right)_{T_{l}} \\
\left(x_{1}^{2}, 1\right)_{T_{l}} & \left(x_{1}^{2}, x_{1}\right)_{T_{l}} & \ldots & \left(x_{1}^{2}, x_{n}\right)_{T_{l}} & \left(x_{1}^{2}, x_{1}^{2}\right)_{T_{l}} & \ldots & \left(x_{1}^{2}, x_{n}^{k+1}\right)_{T_{l}} \\
\vdots & \vdots & \vdots & \vdots & \vdots & \vdots & \\
\left(x_{n}^{j}, 1\right)_{T_{l}} & \left(x_{n}^{j}, x_{1}\right)_{T_{l}} & \ldots & \left(x_{n}^{j}, x_{n}\right)_{T_{l}} & \left(x_{n}^{j}, x_{1}^{2}\right)_{T_{l}} & \ldots & \left(x_{n}^{j}, x_{n}^{k+1}\right)_{T_{l}}
\end{array}\right)_{C_{j+n}^{j} \times C_{k+1+n}^{n}}
$$

yields $\mathbf{a}=0$.

It is easily seen that a necessary condition for this claim is

$$
n_{i} \times C_{j+n}^{n} \geq C_{k+1+n}^{n} .
$$

In other words, the number of equations in the system $A \mathbf{a}=0$ is greater than or equal to the number of variables.

Remark 3.2. This theorem states that for a given $j$, each patch $M_{i}$ has to contain at least $C_{k+1+n}^{n} / C_{j+n}^{n}$ elements. On the other hand, for larger $j$, the number $C_{k+1+n}^{n} / C_{j+n}^{n}$ becomes smaller. Therefore, it would be natural to choose the largest $j$ such that Condition 3.1 and the projection property (3.3) hold. We refer the reader to Remark 3.1 for the range of $j$ in the case of specific elements.

Since Condition 3.1 depends on the choice of $M_{i} \in \mathbb{M}_{h}$, it is not easy to provide general recommendations for its verification. However, for certain structured meshes with all patches $M_{1}, M_{2}, \ldots, M_{n_{h}}$ having the same number of elements, the verification of this condition on each $M_{i}$ can be done on a reference patch $\hat{M}$. To this end, we assume that

$$
M_{i}=\bigcup_{l=1}^{n_{i}} T_{l}, \quad \hat{M}=\bigcup_{l=1}^{n_{i}} \hat{T}_{l},
$$


where $T_{l}=\Psi_{l}\left(\hat{T}_{l}\right)$, the function $\Psi_{l}: \mathbf{R}^{n} \rightarrow \mathbf{R}^{n}$ is defined by

$$
\Psi(\hat{\mathbf{x}}):=\mathbf{B}_{l} \hat{\mathbf{x}}+\mathbf{b}_{l},
$$

the matrix $\mathbf{B}_{l} \in \mathbf{R}^{n \times n}$ is invertible and $\mathbf{b} \in \mathbf{R}^{n}$. For any $v \in L^{2}\left(M_{i}\right)$, we define $\hat{v}(\hat{\mathbf{x}}):=$ $v(\Psi(\hat{\mathbf{x}}))$.

Theorem 3.3. Assume that the conditions (3.11) hold and

$$
\|\hat{q}\|_{0, \hat{M}}^{2} \lesssim\left\|P_{\hat{M}}^{j} \hat{q}\right\|_{0, \hat{M}}^{2} \quad \text { for any } \quad \hat{q} \in \mathscr{P}_{k+1}(\hat{M}) .
$$

Then

$$
\|q\|_{0, M_{i}}^{2} \lesssim\left\|P_{M_{i}}^{j} q\right\|_{0, M_{i}}^{2} \text { for any } q \in \mathscr{P}_{k+1}\left(M_{i}\right)
$$

Proof. It follows from the definitions of the projections $P_{\widehat{T}}^{j}$ and $\widehat{P_{T}^{j}}$ that for any $T=$ $T_{l}, \hat{T}=\hat{T}_{l}, v \in L^{2}(T)$ we have

$$
\left(P_{\hat{T}}^{j} \hat{v}, \hat{w}\right)_{\hat{T}}=(\hat{v}, \hat{w})_{\hat{T}}=(v, w)_{T}=\left(P_{T}^{j} v, w\right)_{T}=\left(\widehat{P_{T}^{j}} v, \hat{w}\right)_{\hat{T}} \quad \text { for all } \quad w \in \mathscr{P}_{j}(T)
$$

Therefore,

$$
P_{\hat{T}}^{j} \hat{v}=\widehat{P_{T}^{j} v}
$$

This and the condition (3.12) yield that for any $q \in \mathscr{P}_{k+1}\left(M_{i}\right)$, we have

$$
\begin{aligned}
\|q\|_{0, M_{i}}^{2} & =\sum_{l=1}^{n_{i}}\|q\|_{0, T_{l}}^{2}=\sum_{l=1}^{n_{i}}\|\hat{q}\|_{0, \hat{T}_{l}}^{2}=\|\hat{q}\|_{0, \hat{M}}^{2} \\
& \lesssim\left\|P_{\hat{M}}^{j} \hat{q}\right\|_{0, \hat{M}}^{2}=\sum_{l=1}^{n_{i}}\left\|P_{\hat{T}_{l}}^{j} \hat{q}\right\|_{0, \hat{T}_{l}}^{2}=\sum_{l=1}^{n_{i}}\left\|\widehat{P_{T_{l}}^{j} q}\right\|_{0, \hat{T}_{l}}^{2} \\
& =\sum_{l=1}^{n_{i}}\left\|P_{T_{l}}^{j} q\right\|_{0, T_{l}}^{2}=\left\|P_{M_{i}}^{j} q\right\|_{0, M_{i}}^{2},
\end{aligned}
$$

and (3.13) is proved.

Remark 3.3. Since $T_{l}=\Psi_{l}\left(\hat{T}_{l}\right), l=1,2, \ldots, n_{i}$, this theorem can be used in the case of structured simplicial meshes or parallelogram/parallelepiped meshes.

As an example, we verify the condition (3.12) with $k=1$ and $j=1$ for rectangular meshes - i.e.

$$
\|\hat{q}\|_{0, \hat{M}}^{2} \lesssim\left\|P_{\hat{M}}^{1} \hat{q}\right\|_{0, \hat{M}}^{2} \quad \text { for all } \quad \hat{q} \in \mathscr{P}_{2}(\hat{M}),
$$

where the reference patch $\hat{M}$ is the square $[-1,1] \times[-1,1]-$ cf. Fig. 1 , which consists of four reference rectangles $\hat{T}_{i}, i=1,2,3,4-$ cf. Fig. 2 . For $\hat{q} \in \mathscr{P}_{2}(\hat{M})$, we assume that

$$
\begin{aligned}
\hat{q} & =a_{1} \hat{x}^{2}+a_{2} \hat{y}^{2}+a_{3} \hat{x}+a_{4} \hat{y}+a_{5} \hat{x} \hat{y}+a_{6}, \\
P_{\hat{T}_{i}}^{1} \hat{q} & =\mathscr{A}_{i} \hat{x}+\mathscr{B}_{i} \hat{y}+\mathscr{C}_{i},
\end{aligned}
$$



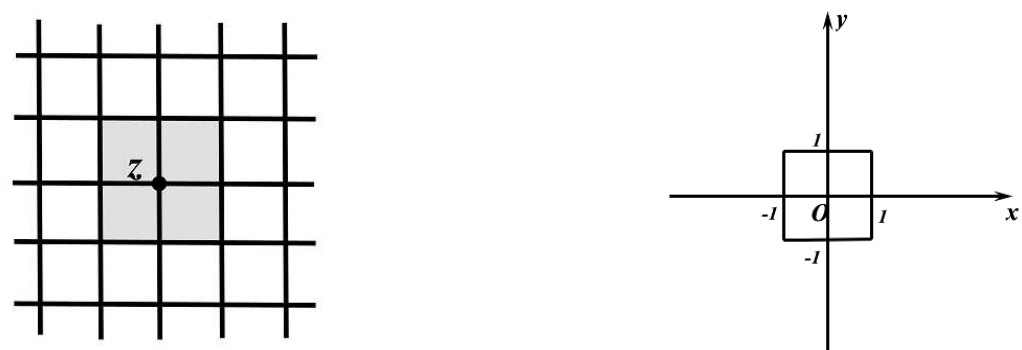

Figure 1: Rectangular mesh, $k=1, j=1$. Left: patch $M$ with respect to node $z$. Right: reference patch $\hat{M}$.
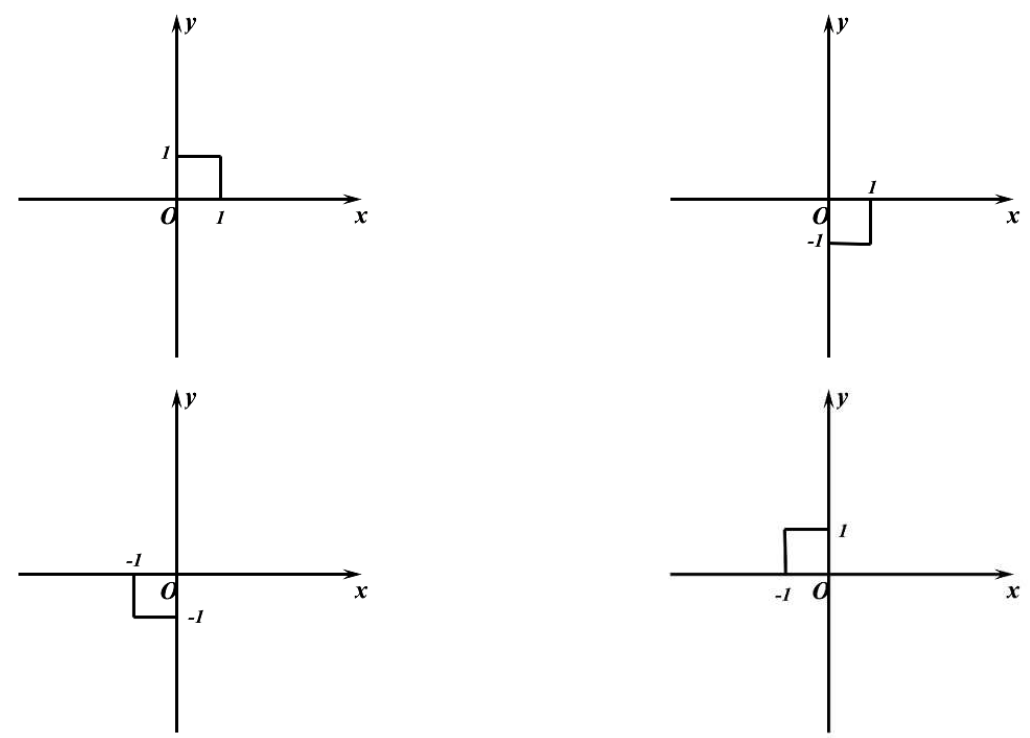

Figure 2: Subelements $\hat{T}_{i}, i=1,2,3,4$ in reference patch $\hat{M}$.

where $a_{l}, \mathscr{A}_{i}, \mathscr{B}_{i}, \mathscr{C}_{i}, l=1,2, \ldots, 6$ and $i=1,2, \ldots, 4$ are constants.

According to the definition of the projection $P_{\hat{M}}^{1}$, we have

$$
(\hat{q}, \hat{x})_{\hat{T}_{i}}=\left(P_{\hat{T}_{i}}^{1} \hat{q}, \hat{x}\right)_{\hat{T}_{i}}, \quad(\hat{q}, \hat{y})_{\hat{T}_{i}}=\left(P_{\hat{T}_{i}}^{1} \hat{q}, \hat{y}\right)_{\hat{T}_{i}}, \quad(\hat{q}, 1)_{\hat{T}_{i}}=\left(P_{\hat{T}_{i}}^{1} \hat{q}, 1\right)_{\hat{T}_{i}}, \quad i=1,2, \ldots, 4,
$$

and simple calculations show that

$$
\begin{array}{llll}
\mathscr{A}_{1}=a_{1}+a_{3}+\frac{1}{2} a_{5}, & \mathscr{B}_{1}=a_{2}+a_{4}+\frac{1}{2} a_{5}, & \mathscr{C}_{1}=-\frac{1}{6} a_{1}-\frac{1}{6} a_{2}-\frac{1}{4} a_{5}+a_{6}, \\
\mathscr{A}_{2}=a_{1}+a_{3}-\frac{1}{2} a_{5}, & \mathscr{B}_{2}=-a_{2}+a_{4}+\frac{1}{2} a_{5}, & \mathscr{C}_{2}=-\frac{1}{6} a_{1}-\frac{1}{6} a_{2}-\frac{1}{4} a_{5}+a_{6}, \\
\mathscr{A}_{3}=-a_{1}+a_{3}+\frac{1}{2} a_{5}, & \mathscr{B}_{3}=a_{2}+a_{4}-\frac{1}{2} a_{5}, & \mathscr{C}_{3}=-\frac{1}{6} a_{1}-\frac{1}{6} a_{2}-\frac{1}{4} a_{5}+a_{6}, \\
\mathscr{A}_{4}=-a_{1}+a_{3}-\frac{1}{2} a_{5}, & \mathscr{B}_{4}=-a_{2}+a_{4}-\frac{1}{2} a_{5}, & \mathscr{C}_{4}=-\frac{1}{6} a_{1}-\frac{1}{6} a_{2}-\frac{1}{4} a_{5}+a_{6} .
\end{array}
$$


Therefore,

$$
\begin{aligned}
\left\|P_{\hat{M}}^{1} \hat{q}\right\|_{0, \hat{M}}^{2}= & \sum_{i=1}^{4}\left\|P_{\hat{T}_{i}}^{1} \hat{q}\right\|_{0, \hat{T}_{i}}^{2}=\frac{1}{3} \mathscr{A}_{1}^{2}+\frac{1}{3} \mathscr{B}_{1}^{2}+\mathscr{C}_{1}^{2}+\frac{1}{2} \mathscr{A}_{1} \mathscr{B}_{1}+\mathscr{B}_{1} \mathscr{C}_{1}+\mathscr{A}_{1} \mathscr{C}_{1} \\
& +\frac{1}{3} \mathscr{A}_{2}^{2}+\frac{1}{3} \mathscr{B}_{2}^{2}+\mathscr{C}_{2}^{2}-\frac{1}{2} \mathscr{A}_{2} \mathscr{B}_{2}-\mathscr{B}_{2} \mathscr{C}_{2}+\mathscr{A}_{2} \mathscr{C}_{2} \\
& +\frac{1}{3} \mathscr{A}_{3}^{2}+\frac{1}{3} \mathscr{B}_{3}^{2}+\mathscr{C}_{3}^{2}-\frac{1}{2} \mathscr{A}_{3} \mathscr{B}_{3}+\mathscr{B}_{3} \mathscr{C}_{3}-\mathscr{A}_{3} \mathscr{C}_{3} \\
& +\frac{1}{3} \mathscr{A}_{4}^{2}+\frac{1}{3} \mathscr{B}_{4}^{2}+\mathscr{C}_{4}^{2}+\frac{1}{2} \mathscr{A}_{4} \mathscr{B}_{4}-\mathscr{B}_{4} \mathscr{C}_{4}-\mathscr{A}_{4} \mathscr{C}_{4} \\
= & \frac{1}{3} a_{1}^{2}+\frac{1}{3} a_{2}^{2}+\frac{4}{3} a_{3}^{2}+\frac{4}{3} a_{4}^{2}+\frac{5}{12} a_{5}^{2}+\left(\frac{1}{3} a_{1}+\frac{1}{3} a_{2}-a_{5}+a_{6}\right)^{2} \\
& +\frac{1}{3}\left(a_{1}+a_{2}+3 a_{6}\right)^{2} . \\
&
\end{aligned}
$$

On the other hand,

$$
\|\hat{w}\|_{0, \hat{M}}^{2}=\frac{16}{45} a_{1}^{2}+\frac{16}{45} a_{2}^{2}+\frac{4}{3} a_{3}^{2}+\frac{4}{3} a_{4}^{2}+\frac{4}{9} a_{5}^{2}+\frac{4}{9}\left(a_{1}+a_{2}+3 a_{6}\right)^{2},
$$

so that

$$
\|\hat{q}\|_{\hat{M}}^{2} \leq 2\left\|P_{T}^{j} \hat{q}\right\|_{\hat{M}}^{2}
$$

\section{Numerical Results}

In this section, we apply the proposed post-processing method to the triangular elements $\mathbf{R T}_{k}, \mathbf{B D M}_{k}, \mathbf{H D G}_{k}$ and to the rectangular elements $\mathbf{R T}_{[k]}, \mathbf{B D M}_{[k]}, \mathbf{H D G}_{[k]}$. To this end, we consider the following second order elliptic equations:

$$
\begin{array}{ll}
\mathbf{q}+\nabla u=0 & \text { in } \Omega, \\
\nabla \cdot \mathbf{q}=f & \text { in } \Omega, \\
u=g & \text { on } \partial \Omega,
\end{array}
$$

where $\Omega \subset \mathbb{R}^{2}$ is a bounded polyhedral domain, $f \in L^{2}(\Omega)$ and $g \in H^{1 / 2}(\partial \Omega)$.

For simplicity, we follow the HDG framework of [12] to describe the finite element schemes considered. Let $\mathscr{T}_{h}:=\bigcup\{T\}$ be a conforming and shape regular partition of $\Omega$, where each $T$ is a polyhedral element. Denote by $F_{h}:=\bigcup\{F\}$ the set of all edges/faces of all $T \in \mathscr{T}_{h}$, and let $\partial \mathscr{T}_{h}:=\left\{\partial T: T \in \mathscr{T}_{h}\right\}$. We consider the local finite dimensional spaces $\mathrm{V}(T), W(T)$ and $\widetilde{W}(F)$ and set

$$
\begin{aligned}
\mathbf{V}_{h}:= & \left\{\mathbf{v} \in \mathbf{L}^{2}\left(\mathscr{T}_{h}\right):\left.\mathbf{v}\right|_{T} \in \mathbf{V}(T) \text { for any } T \in \mathscr{T}_{h}\right\}, \\
W_{h}:= & \left\{w \in L^{2}\left(\mathscr{T}_{h}\right):\left.w\right|_{T} \in W(T) \text { for any } T \in \mathscr{T}_{h}\right\}, \\
\widetilde{W}_{h}(g):= & \left\{\mu \in L^{2}\left(\mathscr{T}_{h}\right):\left.\mu\right|_{F} \in \widetilde{W}(F),(\mu, \tilde{\mu})_{F \cap \partial \Omega}=(g, \tilde{\mu})_{F \cap \partial \Omega}\right. \\
& \text { for any } \left.F \in F_{h}, \forall \tilde{\mu} \in \widetilde{W}(F)\right\} .
\end{aligned}
$$


Notice that

$$
\widetilde{W}_{h}(0)=\left\{\mu \in L^{2}\left(\mathscr{T}_{h}\right):\left.\mu\right|_{F} \in \widetilde{W}(F) \text { for all } F \in F_{h} \text { and }\left.\mu\right|_{\partial \Omega}=0\right\} .
$$

The HDG method for the problem (4.1) consists in finding $\left(u_{h}, \mathbf{q}_{h}, \hat{u}_{h}\right) \in W_{h} \times \mathbf{V}_{h} \times \widetilde{W}_{h}(g)$ such that

$$
\begin{array}{ll}
\left(\mathbf{q}_{h}, \mathbf{v}\right)_{\mathscr{T}_{h}}-\left(u_{h}, \nabla \cdot \mathbf{v}\right)_{\mathscr{T}_{h}}+\left\langle\hat{u}_{h}, \mathbf{v} \cdot \mathbf{n}\right\rangle_{\partial \mathscr{T}_{h}}=0 & \text { for any } \mathbf{v} \in \mathbf{V}_{h}, \\
\left(w, \nabla \cdot \mathbf{q}_{h}\right)_{\mathscr{T}_{h}}+\left\langle\alpha\left(u_{h}-\hat{u}_{h}\right), w\right\rangle_{\partial \mathscr{T}_{h}}=(f, w)_{\partial \mathscr{T}_{h}} & \text { for any } w \in W_{h}, \\
\left\langle\mathbf{q}_{h} \cdot \mathbf{n}+\alpha\left(u_{h}-\hat{u}_{h}\right), \mu\right\rangle_{\partial \mathscr{T}_{h}}=0 & \text { for any } \mu \in \widetilde{W}_{h}(0),
\end{array}
$$

where

$$
(\cdot, \cdot)_{\mathscr{T}_{h}}:=\sum_{T \in \mathscr{T}_{h}}(\cdot, \cdot)_{T}, \quad\langle\cdot, \cdot\rangle_{\partial \mathscr{T}_{h}}:=\sum_{T \in \mathscr{T}_{h}}(\cdot, \cdot)_{\partial T}
$$

and $\alpha$ is a nonnegative penalty function defined on $\partial \mathscr{T}_{h}$.

Within the framework (4.2), there are elements of six types - viz. the hybridised versions of $\mathbf{R T}_{k}, \mathbf{R T}_{[k]}, \mathbf{B D M}_{k}, \mathbf{B D M}_{[k]}$ and the hybridised discontinuous Galerkin elements $\mathrm{HDG}_{k}, \mathrm{HDG}_{[k]}$, which respectively correspond to the following choices of local spaces $\mathbf{V}(T), W(T)$ and $\widetilde{W}(F)$ and penalty functions $\alpha$ :

- Hybridised $\mathbf{R T}_{k}$ triangular elements: $k \geq 0, \mathbf{V}(T)=\mathscr{P}_{k}(T)^{2}+\mathscr{P}_{k}(T) x, W(T)=$ $\mathscr{P}_{k}(T), \widetilde{W}(F)=\mathscr{P}_{k}(F)$ and $\alpha=0$. Here and in the following $\boldsymbol{x}=(x, y)^{t}$.

- Hybridised $\mathbf{R T}_{[k]}$ rectangular elements: $k \geq 0, \mathbf{V}(T)=\mathscr{P}_{k}(T)^{2}+\mathscr{P}_{k}(T) \boldsymbol{x}, W(T)=$ $\mathscr{Q}_{k}(T), \widetilde{W}(F)=\mathscr{P}_{k}(F)$ and $\alpha=0$.

- Hybridised $\mathbf{B D M}_{k}$ triangular elements: $k \geq 2, \mathbf{V}(T)=\mathscr{P}_{k}(T)^{2}, W(T)=\mathscr{P}_{k-1}(T)$, $\widetilde{W}(F)=\mathscr{P}_{k}(F)$ and $\alpha=0$.

- Hybridised $\mathbf{B D M}_{[k]}$ rectangular elements: $k \geq 2, \mathrm{~V}(T)=\mathscr{P}_{k}(T)^{2}+\nabla \times\left(x y x^{k}\right)+\nabla \times$ $\left(x y y^{k}\right), W(T)=\mathscr{P}_{k-1}(T), \widetilde{W}(F)=\mathscr{P}_{k}(F)$ and $\alpha=0$.

- HDG $_{k}$ triangular elements: $k \geq 1, \mathbf{V}(T)=\mathscr{P}_{k}(T)^{2}, W(T)=\mathscr{P}_{k}(T), \widetilde{W}(F)=\mathscr{P}_{k}(F)$ and $\alpha=1 / h_{T}$.

- HDG $_{[k]}$ rectangular elements: $k \geq 1, \mathbf{V}(T)=\mathscr{P}_{k}(T)^{2}+\nabla \times\left(x y \overline{\mathscr{P}}_{k}(T)\right), W(T)=$ $\mathscr{P}_{k}(T), \widetilde{W}(F)=\mathscr{P}_{k}(F)$, and $\alpha=1 / h_{T}$. Here $\overline{\mathscr{P}}_{k}(T)$ is the set of all homogeneous polynomials on $T$ of the degree at most $k$.

We recall that the hybridised RT elements and hybridised BDM elements are equivalent to the corresponding RT and BDM mixed elements, respectively $[1,8]$.

Let $\Omega=(0,1) \times(0,1)$ and $f$ and $g$ be functions such that the function

$$
u=\sin (\pi x) \cdot \sin (\pi y)
$$

is the solution of the model problem (4.1). 
We compute the hybridised $\mathbf{R T}_{k}$ and $\mathbf{R T}_{[k]}$ elements for $k=0,1,2$, the hybridised $\mathbf{B D M}_{k}$ and $\mathbf{B D M}_{[k]}$ elements for $k=2,3$, and the $\mathbf{H D G}_{k}$ and $\mathbf{H D G}_{[k]}$ elements for $k=1,2$ on the $N \times N$ uniform meshes with $N=4,8,16,32-$ cf. Fig. 3 .
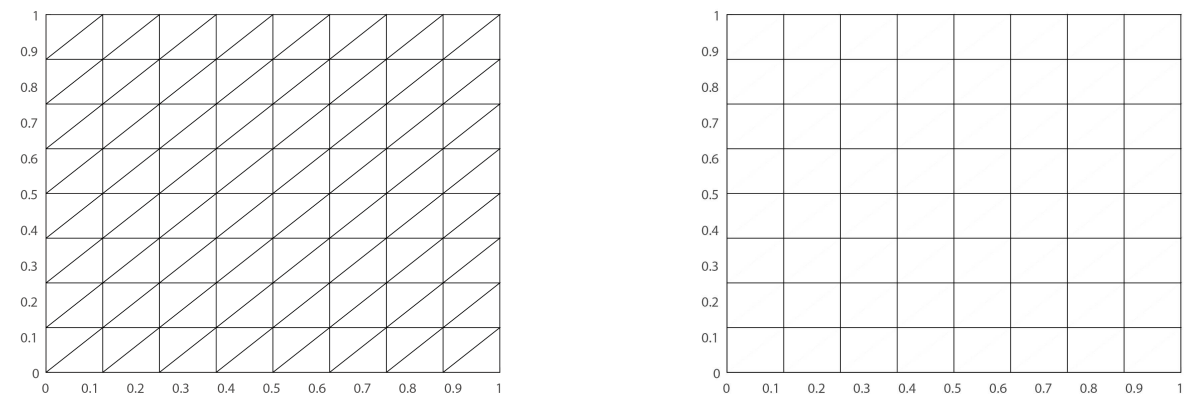

Figure 3: $8 \times 8$ uniform triangular and rectangular meshes.

Fig. 4 demonstrates the patch choice for an interior or a boundary node $z=\boldsymbol{x}_{i}$ with the corresponding $M_{i}$ consisting of shadow elements. If $j=k$ and $j=k-1$, we choose $M_{i}$ as in Figs. 4(a)-4(c) for triangular meshes and as in Figs. 4(g)-4(h) for rectangular meshes. Although it was recommended in Theorem 3.2 and Remark 3.2 to choose the largest $j$ such that for a given $k$ Condition 3.1 and the projection property (3.3) hold, a smaller $j$ also works well in the post-processing method proposed. To show this, we also consider $\mathbf{R T}_{k}$ for $k=2, j=0$. Figs. 4(d)-4(e) show possible choices of $M_{i}$ in this situation.

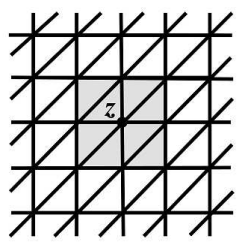

a)

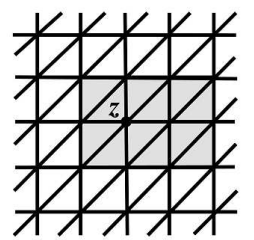

d)

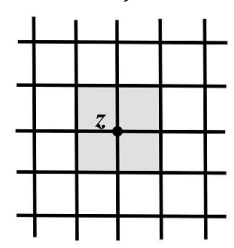

g)

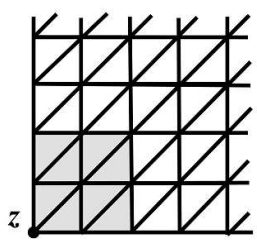

b)

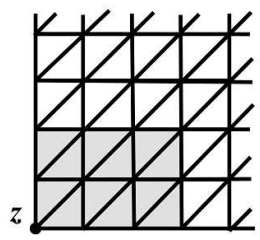

e)

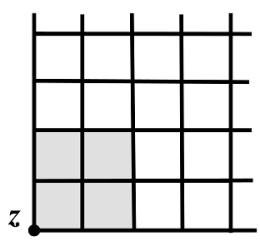

h)

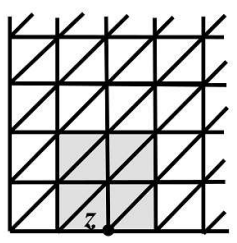

c)

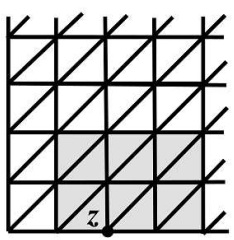

f)

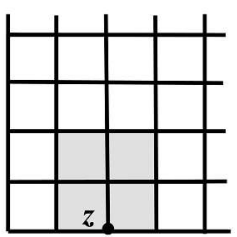

i)

Figure 4: Element selection for $M_{i}$. 
Tables 1-7 provide numerical relative errors $\left\|u-u_{h}\right\|_{0},\left\|\widetilde{P}_{h} u-u_{h}\right\|_{0}$, and $\left\|u-u_{h}^{*}\right\|_{0}$ for the elements $\mathbf{R T}_{k}, \mathbf{R T}_{[k]}, \mathbf{B D M}_{k}, \mathbf{B D M}_{[k]}, \mathbf{H D G}_{k}$, and $\mathbf{H D G}_{[k]}$. In particular, we want to point out the following features:

- $\left\|\widetilde{P}_{h} u-u_{h}\right\|_{0}$ has the convergence order $k+2$ for all elements, which satisfy the superconvergence estimate (3.7).

- The corresponding post-processing solution $u_{h}^{*}$ is of higher accuracy than the finite element solution $u_{h}$. More precisely, $\left\|u-u_{h}^{*}\right\|_{0}$ has the same convergence order $k+2$ as $\left\|\widetilde{P}_{h} u-u_{h}\right\|_{0}$, consistent with Theorem 3.1.

Table 1: Convergence history for $\mathbf{R T}_{k}$ triangular elements, $j=k$.

\begin{tabular}{||c|c|cccccc||}
\hline \multirow{2}{*}{ Degree $k$} & \multirow{2}{*}{ Mesh } & $\frac{\left\|u-u_{h}\right\|_{0}}{\|u\|_{0}}$ & & $\frac{\left\|\widetilde{P}_{h} u-u_{h}\right\|_{0}}{\|u\|_{0}}$ & & $\frac{\left\|u-u_{h}^{*}\right\|_{0}}{\|u\|_{0}}$ & \\
\cline { 3 - 8 } & & Error & Order & Error & Order & Error & Order \\
\hline 0 & $4 \times 4$ & $2.57 \mathrm{E}-01$ & - & $1.66 \mathrm{E}-02$ & - & $8.24 \mathrm{E}-02$ & - \\
& $8 \times 8$ & $1.30 \mathrm{E}-01$ & 0.98 & $4.50 \mathrm{E}-03$ & 1.88 & $1.92 \mathrm{E}-02$ & 2.10 \\
& $16 \times 16$ & $6.54 \mathrm{E}-02$ & 0.99 & $1.18 \mathrm{E}-03$ & 1.93 & $4.00 \mathrm{E}-03$ & 2.26 \\
& $32 \times 32$ & $3.27 \mathrm{E}-02$ & 1.00 & $2.85 \mathrm{E}-04$ & 2.04 & $8.75 \mathrm{E}-04$ & 2.19 \\
\hline \multirow{3}{*}{1} & $4 \times 4$ & $3.91 \mathrm{E}-02$ & - & $1.80 \mathrm{E}-03$ & - & $2.08 \mathrm{E}-02$ & - \\
& $8 \times 8$ & $9.90 \mathrm{E}-03$ & 1.98 & $2.12 \mathrm{E}-04$ & 3.08 & $2.40 \mathrm{E}-03$ & 3.11 \\
& $16 \times 16$ & $2.50 \mathrm{E}-03$ & 1.99 & $2.61 \mathrm{E}-05$ & 3.02 & $3.19 \mathrm{E}-04$ & 2.90 \\
& $32 \times 32$ & $6.21 \mathrm{E}-04$ & 2.00 & $3.26 \mathrm{E}-06$ & 3.00 & $3.77 \mathrm{E}-05$ & 2.94 \\
\hline 2 & $4 \times 4$ & $4.30 \mathrm{E}-03$ & - & $7.01 \mathrm{E}-04$ & - & $3.50 \mathrm{E}-03$ & - \\
& $8 \times 8$ & $5.49 \mathrm{E}-04$ & 2.97 & $4.53 \mathrm{E}-05$ & 3.94 & $2.56 \mathrm{E}-04$ & 3.77 \\
& $16 \times 16$ & $6.89 \mathrm{E}-05$ & 2.99 & $2.87 \mathrm{E}-06$ & 3.98 & $1.72 \mathrm{E}-05$ & 3.90 \\
& $32 \times 32$ & $8.63 \mathrm{E}-06$ & 3.00 & $1.85 \mathrm{E}-07$ & 3.96 & $1.01 \mathrm{E}-06$ & 3.95 \\
\hline
\end{tabular}

Table 2: Convergence history for $\mathbf{R T}_{k}$ triangular elements, $j \leq k-1$.

\begin{tabular}{||c|c|cccccc||}
\hline \multirow{2}{*}{ Degree $k$} & \multirow{2}{*}{ Mesh } & $\frac{\left\|u-u_{h}\right\|_{0}}{\|u\|_{0}}$ & & $\frac{\left\|u-u_{h}^{*}\right\|_{0}}{\|u\|_{0}}(j=0)$ & \multicolumn{3}{c||}{$\frac{\left\|u-u_{h}^{*}\right\|_{0}}{\|u\|_{0}}(j=1)$} \\
& & Error & Order & Error & Order & Error & Order \\
\cline { 3 - 9 } & $4 \times 4$ & $3.91 \mathrm{E}-02$ & - & $4.27 \mathrm{E}-02$ & - & - & \\
& $8 \times 8$ & $9.90 \mathrm{E}-03$ & 1.98 & $5.81 \mathrm{E}-03$ & 2.91 & - & \\
& $16 \times 16$ & $2.50 \mathrm{E}-03$ & 1.99 & $7.48 \mathrm{E}-04$ & 2.96 & - & \\
& $32 \times 32$ & $6.21 \mathrm{E}-04$ & 2.00 & $9.56 \mathrm{E}-05$ & 2.97 & - & \\
\hline 2 & $4 \times 4$ & $4.30 \mathrm{E}-03$ & - & $4.48 \mathrm{E}-03$ & - & $3.80 \mathrm{E}-03$ & - \\
& $8 \times 8$ & $5.49 \mathrm{E}-04$ & 2.97 & $3.25 \mathrm{E}-04$ & 3.78 & $2.70 \mathrm{E}-04$ & 3.81 \\
& $16 \times 16$ & $6.89 \mathrm{E}-05$ & 2.99 & $2.27 \mathrm{E}-05$ & 3.84 & $1.83 \mathrm{E}-05$ & 3.89 \\
& $32 \times 32$ & $8.63 \mathrm{E}-06$ & 3.00 & $1.54 \mathrm{E}-06$ & 3.88 & $1.18 \mathrm{E}-06$ & 3.95 \\
\hline
\end{tabular}


Table 3: Convergence history for $\mathbf{B D M}_{k}$ triangular elements, $j=k-1$.

\begin{tabular}{||c|c|cccccc||}
\hline \multirow{2}{*}{ Degree $k$} & \multirow{2}{*}{ Mesh } & $\frac{\left\|u-u_{h}\right\|_{0}}{\|u\|_{0}}$ & & $\frac{\left\|\widetilde{P}_{h} u-u_{h}\right\|_{0}}{\|u\|_{0}}$ & & \multicolumn{3}{c||}{$\frac{\left\|u-u_{h}^{*}\right\|_{0}}{\|u\|_{0}}$} & \\
\cline { 3 - 7 } & & Error & Order & Error & Order & Error & Order \\
\hline 2 & $4 \times 4$ & $3.91 \mathrm{E}-02$ & - & $1.70 \mathrm{E}-03$ & - & $1.44 \mathrm{E}-02$ & - \\
& $8 \times 8$ & $9.90 \mathrm{E}-03$ & 1.98 & $1.10 \mathrm{E}-04$ & 3.95 & $8.52 \mathrm{E}-04$ & 4.07 \\
& $16 \times 16$ & $2.50 \mathrm{E}-03$ & 1.99 & $6.94 \mathrm{E}-06$ & 3.98 & $4.00 \mathrm{E}-05$ & 4.41 \\
& $32 \times 32$ & $6.21 \mathrm{E}-04$ & 2.00 & $4.13 \mathrm{E}-07$ & 4.07 & $1.92 \mathrm{E}-06$ & 4.38 \\
\hline 3 & $4 \times 4$ & $4.30 \mathrm{E}-03$ & - & $4.68 \mathrm{E}-05$ & - & $7.70 \mathrm{E}-03$ & - \\
& $8 \times 8$ & $5.49 \mathrm{E}-04$ & 2.97 & $1.67 \mathrm{E}-06$ & 4.80 & $3.21 \mathrm{E}-04$ & 4.58 \\
& $16 \times 16$ & $6.89 \mathrm{E}-05$ & 2.99 & $5.57 \mathrm{E}-08$ & 4.91 & $1.15 \mathrm{E}-05$ & 4.81 \\
& $32 \times 32$ & $8.63 \mathrm{E}-06$ & 3.00 & $1.79 \mathrm{E}-09$ & 4.96 & $3.70 \mathrm{E}-07$ & 4.95 \\
\hline
\end{tabular}

Table 4: Convergence history for $\mathbf{H D G}_{k}$ triangular elements, $j=k-1$.

\begin{tabular}{||c|c|cccccc||}
\hline \multirow{2}{*}{ Degree $k$} & \multirow{2}{*}{ Mesh } & $\frac{\left\|u-u_{h}\right\|_{0}}{\|u\|_{0}}$ & & $\frac{\left\|\widetilde{P}_{h} u-u_{h}\right\|_{0}}{\|u\|_{0}}$ & & \multicolumn{3}{c||}{$\frac{\left\|u-u_{h}^{*}\right\|_{0}}{\|u\|_{0}}$} & \\
\cline { 3 - 7 } & & Error & Order & Error & Order & Error & Order \\
\cline { 3 - 8 } 1 & $4 \times 4$ & $4.55 \mathrm{E}-02$ & - & $2.30 \mathrm{E}-03$ & - & $3.84 \mathrm{E}-02$ & - \\
& $8 \times 8$ & $1.09 \mathrm{E}-02$ & 2.06 & $3.10 \mathrm{E}-04$ & 2.89 & $4.93 \mathrm{E}-03$ & 2.96 \\
& $16 \times 16$ & $2.70 \mathrm{E}-03$ & 2.01 & $3.86 \mathrm{E}-05$ & 3.00 & $6.18 \mathrm{E}-04$ & 2.99 \\
& $32 \times 32$ & $6.70 \mathrm{E}-04$ & 2.01 & $4.83 \mathrm{E}-06$ & 3.00 & $7.76 \mathrm{E}-05$ & 3.00 \\
\hline 2 & $4 \times 4$ & $5.39 \mathrm{E}-03$ & - & $8.36 \mathrm{E}-04$ & - & $4.76 \mathrm{E}-03$ & - \\
& $8 \times 8$ & $6.81 \mathrm{E}-04$ & 2.98 & $5.37 \mathrm{E}-05$ & 3.96 & $3.12 \mathrm{E}-04$ & 3.93 \\
& $16 \times 16$ & $8.60 \mathrm{E}-05$ & 2.98 & $3.42 \mathrm{E}-06$ & 3.97 & $2.02 \mathrm{E}-05$ & 3.94 \\
& $32 \times 32$ & $1.08 \mathrm{E}-06$ & 2.99 & $2.13 \mathrm{E}-07$ & 4.00 & $1.24 \mathrm{E}-06$ & 4.02 \\
\hline
\end{tabular}

Table 5: Convergence history for $\mathbf{R T}_{[k]}$ rectangular elements, $j=k$.

\begin{tabular}{||c|c|cccccc||}
\hline \multirow{2}{*}{ Degree $k$} & \multirow{2}{*}{ Mesh } & $\frac{\left\|u-u_{h}\right\|_{0}}{\|u\|_{0}}$ & & $\frac{\left\|\widetilde{P}_{h} u-u_{h}\right\|_{0}}{\|u\|_{0}}$ & & $\frac{\left\|u-u_{h}^{*}\right\|_{0}}{\|u\|_{0}}$ & \\
\cline { 3 - 7 } & & Error & Order & Error & Order & Error & Order \\
\hline \multirow{2}{*}{0} & $4 \times 4$ & $3.17 \mathrm{E}-01$ & - & $4.73 \mathrm{E}-02$ & - & $1.06 \mathrm{E}-01$ & - \\
& $8 \times 8$ & $1.59 \mathrm{E}-01$ & 0.99 & $1.26 \mathrm{E}-02$ & 1.90 & $1.79 \mathrm{E}-02$ & 2.57 \\
& $16 \times 16$ & $8.01 \mathrm{E}-02$ & 0.99 & $3.20 \mathrm{E}-03$ & 1.97 & $3.90 \mathrm{E}-03$ & 2.19 \\
& $32 \times 32$ & $4.01 \mathrm{E}-02$ & 1.00 & $8.02 \mathrm{E}-04$ & 1.99 & $9.57 \mathrm{E}-04$ & 2.02 \\
\hline \multirow{2}{*}{1} & $4 \times 4$ & $3.22 \mathrm{E}-02$ & - & $5.33 \mathrm{E}-04$ & - & $1.49 \mathrm{E}-02$ & - \\
& $8 \times 8$ & $8.10 \mathrm{E}-03$ & 1.99 & $5.61 \mathrm{E}-05$ & 3.25 & $1.9 \mathrm{E}-03$ & 2.97 \\
& $16 \times 16$ & $2.00 \mathrm{E}-03$ & 2.02 & $6.30 \mathrm{E}-06$ & 3.15 & $2.30 \mathrm{E}-04$ & 3.05 \\
& $32 \times 32$ & $5.08 \mathrm{E}-04$ & 1.98 & $7.60 \mathrm{E}-07$ & 3.06 & $2.75 \mathrm{E}-05$ & 3.06 \\
\hline 2 & $4 \times 4$ & $2.10 \mathrm{E}-03$ & - & $1.41 \mathrm{E}-05$ & - & $1.70 \mathrm{E}-03$ & - \\
& $8 \times 8$ & $2.69 \mathrm{E}-04$ & 2.96 & $7.42 \mathrm{E}-07$ & 4.24 & $9.68 \mathrm{E}-05$ & 4.13 \\
& $16 \times 16$ & $3.41 \mathrm{E}-05$ & 2.98 & $4.12 \mathrm{E}-08$ & 4.17 & $5.98 \mathrm{E}-06$ & 4.02 \\
& $32 \times 32$ & $4.20 \mathrm{E}-06$ & 3.02 & $2.50 \mathrm{E}-9$ & 4.04 & $3.74 \mathrm{E}-07$ & 4.00 \\
\hline
\end{tabular}


Table 6: Convergence history for $\mathbf{B D M}_{[k]}$ rectangular elements, $j=k-1$.

\begin{tabular}{||c|c|cccccc||}
\hline \multirow{2}{*}{ Degree $k$} & \multirow{2}{*}{ Mesh } & $\frac{\left\|u-u_{h}\right\|_{0}}{\|u\|_{0}}$ & & $\frac{\left\|\widetilde{P}_{h} u-u_{h}\right\|_{0}}{\|u\|_{0}}$ & \multicolumn{3}{c||}{$\frac{\left\|u-u_{h}^{*}\right\|_{0}}{\|u\|_{0}}$} \\
\cline { 3 - 8 } & & Error & Order & Error & Order & Error & Order \\
\hline 2 & $4 \times 4$ & $5.94 \mathrm{E}-02$ & - & $1.70 \mathrm{E}-03$ & - & $6.30 \mathrm{E}-03$ & - \\
& $8 \times 8$ & $1.51 \mathrm{E}-02$ & 1.97 & $1.10 \mathrm{E}-04$ & 3.95 & $4.32 \mathrm{E}-04$ & 3.86 \\
& $16 \times 16$ & $3.80 \mathrm{E}-03$ & 1.99 & $6.94 \mathrm{E}-06$ & 3.98 & $2.57 \mathrm{E}-05$ & 4.07 \\
& $32 \times 32$ & $9.50 \mathrm{E}-04$ & 2.00 & $4.13 \mathrm{E}-07$ & 4.07 & $1.53 \mathrm{E}-06$ & 4.07 \\
\hline 3 & $4 \times 4$ & $7.50 \mathrm{E}-03$ & - & $6.68 \mathrm{E}-05$ & - & $1.30 \mathrm{E}-03$ & - \\
& $8 \times 8$ & $9.55 \mathrm{E}-04$ & 2.97 & $1.85 \mathrm{E}-06$ & 5.16 & $2.88 \mathrm{E}-05$ & 5.49 \\
& $16 \times 16$ & $1.20 \mathrm{E}-04$ & 2.99 & $5.14 \mathrm{E}-08$ & 5.17 & $7.45 \mathrm{E}-07$ & 5.27 \\
& $32 \times 32$ & $1.50 \mathrm{E}-05$ & 3.00 & $1.56 \mathrm{E}-09$ & 5.04 & $2.33 \mathrm{E}-08$ & 5.00 \\
\hline
\end{tabular}

Table 7: Convergence history for $\mathrm{HDG}_{[k]}$ rectangular elements, $j=k-1$.

\begin{tabular}{||c|c|cccccc||}
\hline \multirow{2}{*}{ Degree $k$} & \multirow{2}{*}{ Mesh } & $\frac{\left\|u-u_{h}\right\|_{0}}{\|u\|_{0}}$ & & $\frac{\left\|\widetilde{P}_{h} u-u_{h}\right\|_{0}}{\|u\|_{0}}$ & \multicolumn{3}{c||}{$\frac{\left\|u-u_{h}^{*}\right\|_{0}}{\|u\|_{0}}$} \\
\cline { 3 - 8 } & & Error & Order & Error & Order & Error & Order \\
\hline \multirow{2}{*}{1} & $4 \times 4$ & $6.29 \mathrm{E}-02$ & - & $2.52 \mathrm{E}-03$ & - & $3.94 \mathrm{E}-02$ & - \\
& $8 \times 8$ & $1.59 \mathrm{E}-02$ & 1.98 & $3.23 \mathrm{E}-04$ & 2.96 & $5.03 \mathrm{E}-03$ & 2.97 \\
& $16 \times 16$ & $4.01 \mathrm{E}-03$ & 1.99 & $4.08 \mathrm{E}-05$ & 2.98 & $6.31 \mathrm{E}-04$ & 2.99 \\
& $32 \times 32$ & $1.01 \mathrm{E}-04$ & 1.99 & $5.13 \mathrm{E}-06$ & 2.99 & $7.89 \mathrm{E}-05$ & 3.00 \\
\hline 2 & $4 \times 4$ & $8.67 \mathrm{E}-03$ & - & $9.53 \mathrm{E}-04$ & - & $6.76 \mathrm{E}-03$ & - \\
& $8 \times 8$ & $1.10 \mathrm{E}-03$ & 2.97 & $6.25 \mathrm{E}-05$ & 3.93 & $4.43 \mathrm{E}-04$ & 3.93 \\
& $16 \times 16$ & $1.39 \mathrm{E}-04$ & 2.99 & $3.92 \mathrm{E}-06$ & 3.99 & $2.83 \mathrm{E}-05$ & 3.97 \\
& $32 \times 32$ & $1.74 \mathrm{E}-05$ & 2.99 & $2.45 \mathrm{E}-07$ & 4.00 & $1.78 \mathrm{E}-06$ & 3.99 \\
\hline
\end{tabular}

\section{References}

[1] D.N. Arnold, F. Brezzi., Mixed and nonconforming finite element methods: implementation, postprocessing and error estimates, ESAIM Math. Model. Numer. Anal. 19(1), 7-32 (1985).

[2] D.N. Arnold, F. Brezzi and J. Douglas, PEERS: A new mixed finite element for plane elasticity, Japan J. Appl.Math. 1, 347-367 (1984).

[3] D.N. Arnold and R.S. Falk, A new mixed formulation for elasticity, Numer. Math. 53, 13-30 (1988).

[4] I. Babuska and A. Miller, The post-processing approach in the finite element method. Part 1. Calculation of displacements, stresses, and other higher derivatives of the displacements, Internat. J. Numer. Methods Engrg. 20, 1085-1109 (2010).

[5] I. Babuska and A. Miller, The post-processing approach in the finite element method. Part 2: The calculation of stress intensity factors, Internat. J. Numer. Methods Engrg. 20, 1111-1129 (2010).

[6] I. Babuska and A. Miller, The post-processing approach in the finite element method. Part 3: A posteriori error estimates and adaptive mesh selection, Internat. J. Numer. Methods Engrg. 20, 2311-2324 (2010). 
[7] J. H. Bramble and J. Xu, A local post-processing technique for improving the accuracy in mixed finite-element approximations, SIAM J. Numer. Anal. 26, 1267-1275 (1989).

[8] F. Brezzi, J. Douglas and L.D. Marini, Two families of mixed finite elements for second order elliptic problems, Numer. Math. 47, 19-34 (1985).

[9] F. Brezzi and M. Fortin, Mixed and hybrid finite element methods, Springer Series in Computational Mathematics 15, Springer-Verlag (1991).

[10] C. Chen and Y. Huang, High accuracy theory of finite element methods (in Chinese), Hunan Science Press (1995).

[11] G. Chen and X. Xie., A robust Weak Galerkin finite element method for linear elasticity with strong symmetric stresses, Comput. Methods Appl. Math 16, 389-408 (2016).

[12] B. Cockburn, W. Qiu and K. Shi, Conditions for superconvergence of HDG methods for secondorder elliptic problems, Math. Comp. 81, 1327-1353 (2012).

[13] B. Cockburn and K. Shi, Conditions for superconvergence of HDG methods for Stokes flow, Math. Comp. 82(282), 651-671 (2012).

[14] B. Li and Z. Zhang, Analysis of a class of superconvergence patch recovery techniques for linear and bilinear finite elements, Numer. Methods Partial Differential Equations 15, 151-167 (1999).

[15] Q. Lin and N. Yan, Construction and analysis of hign efficient finite elements (in Chinese), Hebei University Press (1996).

[16] Z.C. Shi and M. Wang, Finite element methods, Science Press (2013).

[17] R. Stenberg, A family of mixed finite elements for the elasticity problem, Numer. Math. 53, 513-538 (1988).

[18] R. Stenberg, Postprocessing schemes for some mixed finite elements, ESAIM Math. Model. Numer. Anal. 25, 151-167 (1991).

[19] J. Xu and Z. Zhang, Analysis of recovery type a posteriori error estimators for mildly structured grids, Math. Comp. 73, 1139-1152 (2003).

[20] Z. Zhang and A. Naga, A new finite element gradient recovery method: superconvergence property, SIAM J. Sci. Comput. 26, 1192-1213 (2005).

[21] Z. Zhang, Ultraconvergence of the patch recovery technique II, Math. Comp. 69, 141-158 (2000).

[22] O.C. Zienkiewicz and J. Zhu, The superconvergence patch recovery and a posteriori error estimates. Part 1: The recovery technique, Internat. J. Numer. Methods Engrg. 33, 1331-1364 (1992).

[23] O.C. Zienkiewicz and J. Zhu, The superconvergence patch recovery and a posteriori error estmates. Part 2: Error estmates and adaptivity, Internat. J. Numer. Methods Engrg. 33, 13651382 (1992). 